\title{
Cesarean section rates in the COVID-19 era: False alarms and the safety of the mother and child
}

\author{
Wissam Arab $^{1}$, David Atallah ${ }^{1}$
}

\section{Dear Editor,}

The COVID-19 pandemic is ongoingly affecting numerous pregnant patients around the world. Meanwhile, nationwide lockdowns have drastically limited counseling and followup visits of women during pregnancy. Throughout these difficulties, delivering via the cesarean route is becoming common, both in the infected and non-infected pregnant population. Looking into pregnant women with COVID-19, around $64 \%$ delivered via a cesarean route ${ }^{1}$, with this rate reaching $93 \%$ in China $^{2}$. Due to the relative state of immunosuppression in pregnancy, women might be at an increased risk of severe illness compared to non-pregnant women, after adjusting for age and other confounding factors ${ }^{3}$. Death has occurred in around $0.1 \%$ of the cases, with ICU admissions reaching $3 \%^{4}$. In China, the rate of severe disease was $8 \%^{2}$.

Knowing that the majority of COVID-19 infections in pregnancy occur in the third trimester ${ }^{2,5}$, with $15 \%$ requiring hospital admission ${ }^{4}$, the increased cesarean rate could be partially justified. However, many patients with non-severe disease are being delivered using the $\mathrm{C}$-section route; experts consider that early delivery, even in non-severe cases, as beneficial for the subsequent treatment and outcome of COVID-19 ${ }^{16}$. This has led to an increase in the rate of preterm deliveries (21\%-31\%), of which only few are related to spontaneous preterm labor and preterm premature rupture of membranes, while the rest are thought to be iatrogenic ${ }^{5}$. Preterm and term $\mathrm{C}$-sections are performed due to concerns that excessive ventilation and stress during labor might aggravate the respiratory and pro-inflammatory status accompanying COVID-197. Evidence also showed that maternal oxygenation can be quickly restored by delivery ${ }^{6}$. In parallel, new papers reported placental infection during the COVID-19, leading to placental vascular disease, preeclampsia-like syndrome, fetal growth restriction and higher risks of perinatal death ${ }^{8}$. Fearing these eventual adverse events could add to the increased rate of iatrogenic preterm deliveries using a C-section route. Also, some laboring women are undergoing $\mathrm{C}$-sections due to prophylactic antiplatelets use against COVID-related thromboembolisms, precluding the administration of epidural anaesthesia; not forgetting the fear of vertical transmission in utero, which, even if yet unproven, is usually thought by obstetricians. This risk has been estimated to be around $5 \%$ and peaks at term ${ }^{1,5}$.

In regard to the pregnant population in general, fears of contracting COVID-19 at healthcare centers as well as difficulties in transportation during lockdown periods has contributed to a reluctance in timely referrals to emergency care units. These facts have led to a delay in the management of obstetrical complications and therefore to an increase in stillbirths and preterm deliveries, with a higher risk of $\mathrm{C}$-section in this context ${ }^{9}$. On the other hand, some obstetricians are reluctant when it comes to exposing themselves, trainees and midwives to pregnant patients whose PCR status is unknown, with a tendency towards pursuing $\mathrm{C}$-sections for laboring women who would normally have better chances for delivering vaginally; obstetricians lowered the C-section threshold during COVID-19 pandemic, with the aim of reducing inpatient maternal stays, cross-infection and the use of protective equipment ${ }^{6}$. Not forgetting, some pregnant women, worried about their partners not making it to the birth unit due to lockdowns and the unpredictability of labor, are choosing elective $\mathrm{C}$-section for delivery.

We support the alarm raised in a previous study ${ }^{10}$, and challenge obstetric-led units in their tendency towards C-section. Favoring vaginal birth in infected women is paramount, as it decreases the risk of clinical deterioration, COVID-related thromboembolisms and neonatal morbidity related to iatrogenic preterm deliveries.

\section{AFFILIATION}

1 Department of Obstetrics and Gynecology, Hôtel-Dieu de France University Hospital, Saint-Joseph University, Beirut, Lebanon

\section{CORRESPONDENGE TO}

David Atallah. Department of Obstetrics and Gynecology, Hotel-Dieu de France University Hospital, Saint Joseph University, P.O. Box 116-5137, Beirut, Lebanon. Email: david.atallah@ gmail.com ORCID ID: https:// orcid.org/0000-0003-15375254

\section{KEYWORDS}

COVID-19, cesarean, preterm, vaginal delivery

$\checkmark$

Received: 23 February 2021 Accepted: 25 March 2021 


\section{REFERENGES}

1. Vardhelli V, Pandita A, Pillai A, Badatya SK. Perinatal COVID-19: review of current evidence and practical approach towards prevention and management. Eur J Pediatr. 2021;180(4):1009-1031. doi:10.1007/s00431-020-03866-3

2. Chen L, Li Q, Zheng D, et al. Clinical Characteristics of Pregnant Women with COVID-19 in Wuhan, China. N Engl J Med. 2020;382(25):e100. doi:10.1056/NEJMc2009226

3. Oakes MC, Kernberg AS, Carter EB, et al. Pregnancy as a risk factor for severe coronavirus disease 2019 using standardized clinical criteria. Am J Obstet Gynecol MFM. 2021;3(3):100319. doi:10.1016/j.ajogmf.2021.100319

4. Data on COVID-19 during Pregnancy: Severity of Maternal Illness. Centers for Disease Control and Prevention. Accessed February 22, 2021. https://covid.cdc.gov/covid-data-tracker/\#pregnant-population

5. Della Gatta AN, Rizzo R, Pilu G, Simonazzi G. Coronavirus disease 2019 during pregnancy: a systematic review of reported cases. Am J Obstet Gynecol. 2020;223(1):36-41. doi:10.1016/j.ajog.2020.04.013

6. Qi H, Luo X, Zheng Y, et al. Safe delivery for pregnancies affected by COVID-19. BJOG. 2020;127(8):927-929. doi:10.1111/1471-0528.16231

7. Vouga M, Grobman WA, Baud D. More on Clinical Characteristics of Pregnant Women with Covid-19 in Wuhan, China. N Engl J Med. 2020;383(7):696-697. doi:10.1056/NEJMc2016881

8. Dashraath P, Wong JLJ, Lim MXK, et al. Coronavirus disease 2019 (COVID-19) pandemic and pregnancy. Am J Obstet Gynecol. 2020;222(6):521-531. doi:10.1016/j.ajog.2020.03.021

9. Khalil A, von Dadelszen P, Draycott T, Ugwumadu A, O'Brien P, Magee L. Change in the Incidence of Stillbirth and Preterm Delivery During the COVID-19 Pandemic. JAMA. 2020;324(7):705-706. doi:10.1001/jama.2020.12746

10. Martínez-Perez O, Vouga M, Cruz Melguizo S, et al. Association Between Mode of Delivery Among Pregnant Women With COVID-19 and Maternal and Neonatal Outcomes in Spain. JAMA. 2020;324(3):296-299. doi:10.1001/jama.2020.10125

\section{CONFLICTS OF INTEREST}

The authors have completed and submitted the ICMJE Form for Disclosure of Potential Conflicts of Interest and none was reported.

\section{FUNDING}

There was no source of funding for this research.

\section{PROVENANGE AND PEER REVIEW}

Not commissioned; internally peer reviewed. 